\title{
Analyse des risques sanitaires dans la production de filtres à eau en céramique à Abidjan, Côte d'Ivoire
}

\author{
Mariette Bediakon GOKPEYA ${ }^{1,2^{*}}$, Julie Kouakou SACKOU ${ }^{1,3}$, Jean Stéphane CLAON ${ }^{1,4}$, \\ Beneld Moh Chantal OKA ${ }^{1}$ et Luc Kouakou KOUADIO ${ }^{1,2}$ \\ ${ }^{I}$ Département de Santé Publique, Hydrologie et Toxicologie, UFR des sciences pharmaceutiques et \\ biologiques, Université Félix Houphouët-Boigny ; 22 BP 1306 Abidjan 22, Côte d'Ivoire. \\ ${ }^{2}$ Institut National d'Hygiène Publique, Abidjan, Côte d'Ivoire ; 01 BPV 14 Abidjan 01 \\ ${ }^{3}$ Institut National de Santé Publique ; BP V 47 Abidjan, Côte d'Ivoire. \\ ${ }^{4}$ Laboratoire d'Analyse et de Contrôle de la qualité de l'eau (LACQUE), Office National de l'Eau Potable, \\ Abidjan, Côte d'Ivoire ; 04 BP 42 Abidjan 04. \\ *Auteur correspondant ; E-mail : kemonmariette@gmail.com; Tél: +225 57652442; 23 BP 434 Abidjan 23.
}

\section{RESUME}

L'usage de filtres en céramique pour le traitement de l'eau au point d'utilisation est considéré comme une approche fiable pour garantir la sécurité sanitaire des approvisionnements en eau potable. Aussi l'association N Christ a récemment vulgarisé en Côte d'Ivoire un filtre dénommé FILTRAO ${ }^{\circledR}$. La nécessité d'une assurance qualité de celui-ci nous a conduits à identifier par la démarche HACCP (Hazard Analysis Critical Control Point), les paramètres critiques de son processus de fabrication. L'étude a été menée à Abidjan dans le cadre du processus de délivrance d'un certificat de salubrité pour le FILTRAO ${ }^{\circledR}$. La démarche HACCP a été conduite à l'unité de production du filtre en sept étapes partant de la constitution d'une équipe HACCP à l'identification des points critiques de contrôle (CCP). Il en ressort que l'argile, la sciure de bois et l'eau sont les matières premières utilisées pour la production du FILTRAO ${ }^{\circledR}$. Le procédé de fabrication était de type artisanal et les CCP se situaient aux étapes d'évaluation de la qualité de l'argile, de traitement mécanique des matières premières et de cuisson des pots céramiques. Ces résultats suggèrent que le plan HACCP peut être adopté comme démarche d'assurance qualité pour optimiser l'efficacité et la sécurité du FILTRAO ${ }^{\circledR}$.

(C) 2019 International Formulae Group. All rights reserved

Mots clés: Filtre en céramique, démarche HACCP, assurance qualité.

\section{Health risk analysis in the production of ceramic water filters in Abidjan, Côte d'Ivoire}

\begin{abstract}
The use of ceramic filters for point-of-use water treatment is considered as a reliable approach to ensure the safety of drinking water supplies. So the association N Christ recently popularized in Côte d'Ivoire a filter called FILTRAOß. The need for quality assurance of this one led us to identify by HACCP (Hazard Analysis Critical Control Point) approach, the critical parameters of its manufacturing process. The study was
\end{abstract}


conducted in Abidjan as part of the process of issuing a health certificate for FILTRAOß. The HACCP approach was conducted at the seven-step filter production unit from the establishment of a HACCP team to the identification of Critical Control Points (CCPs). It shows that clay, sawdust and water are the raw materials used for the production of FILTRAO®. The manufacturing process was artisanal and the CCPs were at the stages of evaluating the quality of the clay, the mechanical treatment of the raw materials and the firing of the ceramic pots. These results suggest that the HACCP plan can be adopted as a quality assurance approach to optimize the effectiveness and safety of FILTRAOß.

(C) 2019 International Formulae Group. All rights reserved

Keywords: Ceramic filter, HACCP approach, quality assurance.

\section{INTRODUCTION}

Dans les pays en développement, la sécurité sanitaire des approvisionnements en eau potable demeure un défi majeur de Santé Publique (Bain et al., 2014 Espérance, 2016 ; Njoyim et al., 2016). Annette et collaborateurs (2014) ont montré à l'issu de leur étude portant évaluation de la charge de morbidité attribuable à l'eau, l'hygiène et l'assainissement dans 145 pays en développement; que la contamination de l'eau, le long de la chaîne d'approvisionnement, a été la cause d'environ cinq cent mille cas de décès par maladies diarrhéiques.

L'une des stratégies proposées par l'OMS en vue d'une meilleure gestion de la sécurité sanitaire de l'eau de boisson est le recours à des méthodes efficaces et durables de traitement et de stockage de l'eau au point d'utilisation (OMS, 2011). Diverses approches ont été ainsi testées pour le traitement domestique de l'eau (Elodie, 2014 ; Robert et James, 2015 ; Aminata et al., 2018). De ces méthodes, l'usage des filtres céramiques s'illustre avec de meilleures performances au regard des conclusions de Hunter (2009) suite à une analyse par méta régression portant comparaison de différentes options de traitements domestiques de l'eau.

Aussi, des modèles de filtres céramiques, produits à base de matériaux locaux et souvent revêtus d'argent colloïdal ont été expérimentés avec succès dans certains pays en développement (Danièle et al., 2006 ; Mark et al., 2008 ; Babafemi et Yinusa, 2015). Toutefois, d'autres travaux ont révélé que ces derniers présentaient un faible débit de filtration, une performance non durable et variable d'un filtre à un autre et d'une région à une autre (Moubokounou, 2010 ; Murphy et al., 2010; The Ceramics Manufacturing Working Group, 2010; Erhuanga et al., 2014).

En Côte d'Ivoire, l'association N'Christ a récemment vulgarisé un filtre à eau en céramique dénommé FILTRAO ${ }^{\circledR}$. Des essais conduits au laboratoire ont montré que celui-ci améliore la qualité organoleptique et microbiologique de l'eau mais présente un faible débit de filtration et un manque de sécurité vis-à-vis de l'eau filtrée par le relargage de substances chimiques notamment les nitrates, les nitrites et les fluorures.

L'utilisation de ces filtres en céramique étant censée répondre à un enjeu de santé publique (améliorer l'accès à l'eau potable), la garantie de leur assurance qualité est cruciale pour éviter de nouveaux risques sanitaires susceptibles d'être liés à leur usage. Cela implique d'optimiser la fiabilité de ces dispositifs par l'adoption de mesures de maîtrise des risques sanitaires applicables à toutes les étapes de la chaîne de production.

L'analyse des risques et identification des points critiques pour leur maitrise, traduit de l'anglais HACCP (Hazard Analysis Critical Control Point) est une démarche fiable visant à garantir l'assurance de la qualité des produits finis. Initialement utilisée dans l'industrie agroalimentaire, l'approche a été adaptée avec succès au domaine du traitement de l'eau potable (Dewettinck et al., 2001 ; Jagals et Jagals, 2004). Ceci suggèrent une possible application de la démarche HACCP au management de la sécurité sanitaire des dispositifs de traitement domestique de l'eau que sont les filtres à eau en céramiques. 
L'objectif de cette étude était d'identifier par la démarche HACCP, les paramètres critiques $\mathrm{du}$ processus de fabrication du FILTRAO ${ }^{\circledR}$.

\section{MATERIEL ET METHODES Cadre de l'étude}

Cette étude a été réalisée dans le cadre du processus de délivrance d'un certificat de salubrité pour le FILTRAO $^{\circledR}$, filtre en céramique produit et commercialisé en Côte d'Ivoire par l'association N'Christ. Il s'agissait de la mise en place d'une démarche qualité à l'unité de production du filtre située à Abidjan au sud du pays. L'étude a été conduite sous la supervision d'une équipe du Laboratoire d'Hygiène de l'Institut National d'Hygiène publique d'Abidjan (INHP). Les principes de l'approche d'analyse des risques et identification des points critiques pour leur maîtrise (HACCP) ont été utilisés pour identifier les potentiels dangers pouvant être liés au processus de fabrication du filtre.

\section{Application des principes HACCP à la production du FILTRAO ${ }^{\circledR}$}

Selon les spécifications du Codex Alimentarius (2003), la démarche HACCP est fondée sur 7 principes avec une mise en place en 12 étapes.

Ce travail étant une étude préliminaire initiée par une équipe externe au personnel de l'unité de production du filtre et visant l'analyse des risques liés à la production de celui-ci, seules les 7 premières étapes du processus HACCP ont été réalisées de la manière suivante :

\section{Mise en place d'une équipe HACCP}

L'équipe constituée pour la conduite de la démarche comprenait le superviseur de la production du filtre en céramique et un stagiaire venu du Laboratoire d'Hygiène de l'INHP.

\section{Description du produit}

Cette étape consiste à décrire tous les paramètres essentiels à l'obtention du produit fini. Dans le cas spécifique de cette étude, les informations relatives aux matières premières, aux conditions de production, aux caractéristiques générales, propriétés physicochimiques et présentation du filtre prêt à l'emploi ont été rassemblées.

\section{Détermination de l'utilisation prévue}

Il s'agissait de définir l'usage auquel est destiné le produit en fonction de l'utilisateur final (Boudjelti, 2017). Au cours de cette étape nous avons identifié la durée de vie, les modalités d'utilisation attendue du produit et examiné l'adéquation entre le produit et les instructions d'utilisation.

\section{Etablissement et vérification du diagramme de fabrication}

Le diagramme de fabrication est la colonne vertébrale de la démarche HACCP (Amroun, 2017). Il s'agissait ici de suivre toutes les étapes du processus de fabrication $\mathrm{du}$ filtre puis de schématiser cet ensemble sous la forme d'un diagramme. Ce dernier a été par la suite vérifié sur le terrain pour prendre en compte d'éventuelles insuffisances et lacunes.

\section{Analyse des dangers}

L'analyse des dangers a été conduite en 2 phases successives. Dans un premier temps, tous les dangers potentiels liés aux étapes de la production du filtre ont été identifiés avec leurs causes et possibles effets. Par la suite les mesures préventives de maîtrise adaptées à chaque danger ont été déterminées.

\section{Identification des points critiques de contrôle (CCP)}

Les CCP sont les étapes du processus pour lesquelles des mesures particulières de surveillance sont nécessaires en complément des mesures préventives. Au moyen d'un arbre HACCP (Figure 1) de décision (Codex Alimentarius, 2003), les points critiques pour la maitrise des risques (CCP) ont été déterminés au cours de cette étude. 


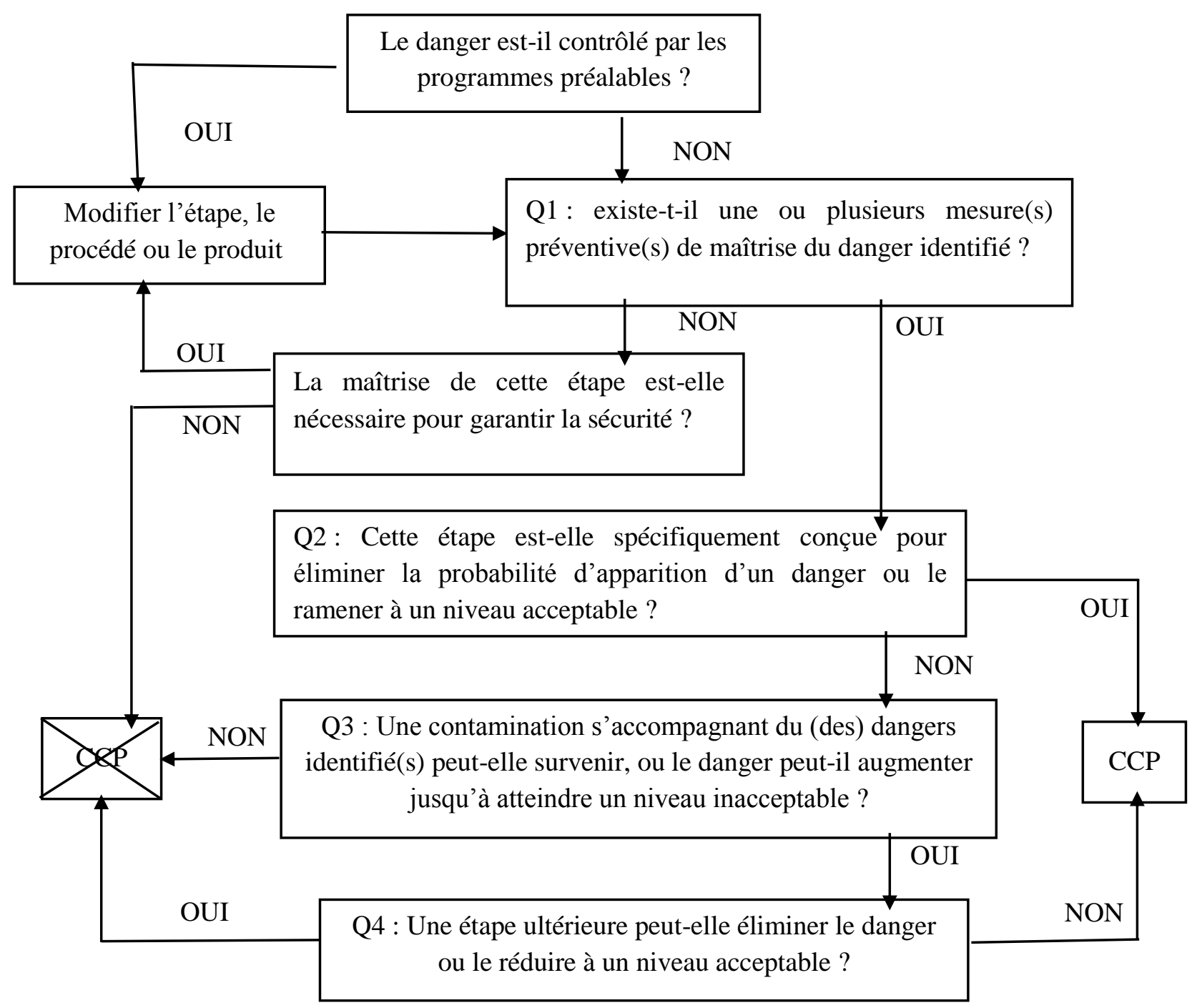

Figure 1 : Arbre HACCP de décision pour l'identification des CCP.

RESULTATS

Description du produit et de son utilisation prévue

Le FILTRAO $^{\circledR}$ (Figure 2) est un filtre à eau en céramique de la forme d'un pot de fleur. Il est Composé de terre cuite poreuse et imprégné de solution de colloïde d'argent. Le dispositif de filtration comprend un pot d'argile associé à un seau en plastique muni d'un robinet. Le Tableau 1 résume la description des matières premières et du produit fini.

\section{Etablissement du diagramme de fabrication}

La production des filtres en céramique FILTRAO ${ }^{\circledR}$ fait appel à une succession d'opérations élémentaires regroupées dans le diagramme de fabrication illustré à la Figure 3. Cette production se fait en 14 étapes pouvant être subdivisées en 3 principales phases à savoir la phase de préparation de la matière première (PHASE I) qui part du choix de l'argile jusqu'à son transport au lieu de production des filtres ; la phase en pré cuisson (PHASE II) qui comprend essentiellement le traitement mécanique de la matière première après réception puis la cuisson au four à feu de bois des pots céramiques; enfin la phase après cuisson (PHASE III) qui regroupe les étapes de refroidissement des pots cuits, d'imprégnation des surfaces au colloïde d'argent et d'assemblage du dispositif final (Figure 3). 


\section{Analyse des dangers}

La description des dangers potentiels et l'analyse des risques associés est une étape déterminante de la démarche HACCP. L'observation de la chaine de production des filtres FILTRAO ${ }^{\circledR}$ révèle l'existence possible de multiples dangers physiques, chimiques et biologiques. Les Tableaux 2 et 3 résument les différents dangers identifiés dans le processus de fabrication du filtre, les sources possibles et les mesures préventives proposées.

\section{Points critiques de contrôle} (CCP) identifiés

L'application des quatre questions de l'arbre HACCP de décision $\left(\mathrm{Q}_{1}, \mathrm{Q}_{2}, \mathrm{Q}_{3}, \mathrm{Q}_{4}\right)$ à chacune des étapes pour lesquelles un danger potentiel a été identifié, nous a permis de déterminer les points critiques de contrôle (Tableau 4). Ces CCP se rencontraient essentiellement aux étapes d'évaluation de la qualité de l'argile, de traitement mécanique des matières premières et de cuisson des pots en céramique.

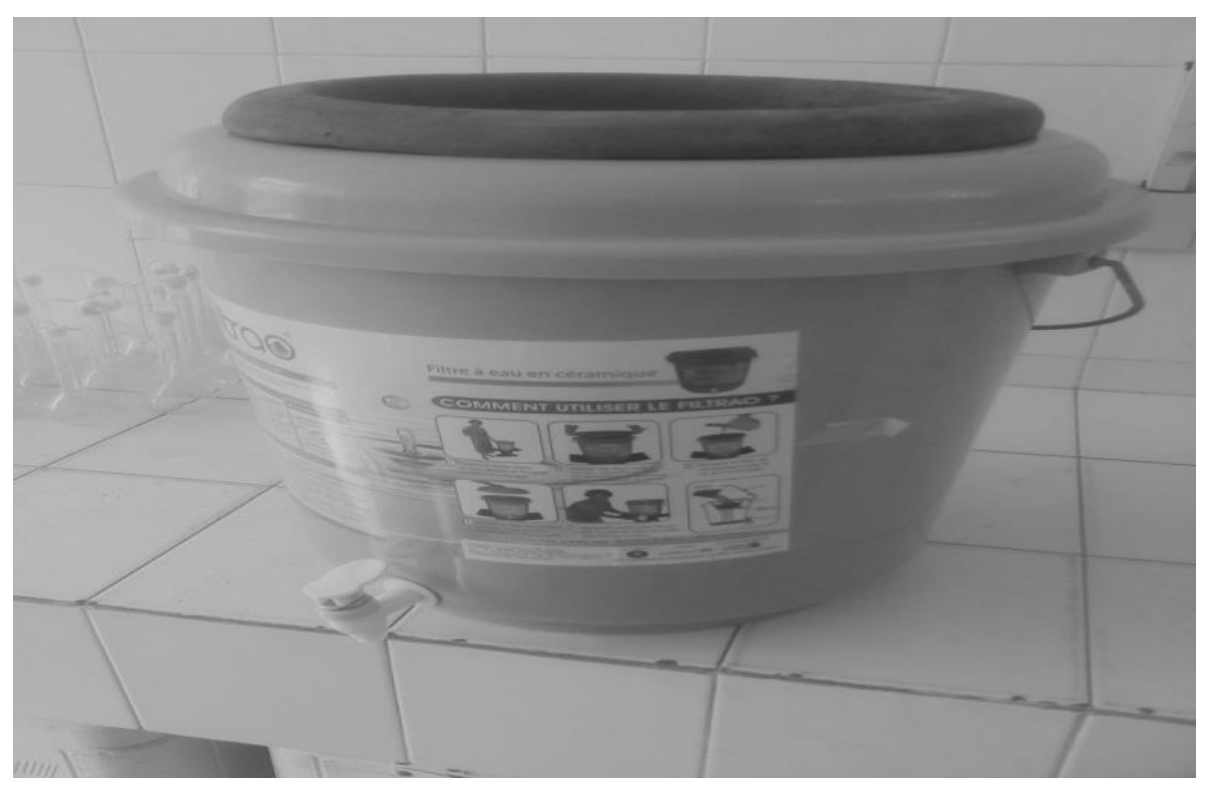

Figure 2 : Filtre en céramique FILTRAO ${ }^{\circledR}$.

Tableau 1: Description du filtre en céramique FILTRAO ${ }^{\circledR}$.

\begin{tabular}{|c|c|}
\hline \multicolumn{2}{|l|}{ Caractéristiques générales } \\
\hline Dénomination & FILTRAO® \\
\hline Nature & Pot en céramique de volume 9 litres \\
\hline Conditionnement & $\begin{array}{l}\text { Sceau en plastique de } 30 \text { litres muni d'un couvercle et d'un } \\
\text { robinet }\end{array}$ \\
\hline Utilisation prévue & Filtration et stockage de l'eau à domicile \\
\hline Durée de vie & 1 à 2 ans \\
\hline \multicolumn{2}{|l|}{ Propriétés physicochimiques } \\
\hline $\mathrm{Ph}$ & Alcalin \\
\hline Potentiel redox & Actif (réduction des nitrates en nitrites) \\
\hline
\end{tabular}


Conservateur Colloïde d'argent

\section{Matières premières}

Poudre d'argile

Sciure de bois

Eau

Traitement

\section{$30 \mathrm{~kg}$ pour un filtre}

8.9 à $10 \mathrm{~kg}$ pour un filtre

12.5 litres pour un filtre

Traitement, préparation et mise en œuvre manuel

Tableau 2 : Principaux dangers identifiés et sources possibles.

\begin{tabular}{|c|c|}
\hline ETAPES DU PROCESSUS & $\begin{array}{l}\text { DANGERS IDENTIFIES ET SOURCES } \\
\text { POSSIBLES }\end{array}$ \\
\hline Choix de l'argile & $\begin{array}{l}\text { - Présence de germes pathogènes (bactéries, } \\
\text { virus, champignons) pouvant résulter de la } \\
\text { contamination du site de prélèvement de } \\
\text { l'argile } \\
\text { - Présence d'impuretés chimiques (métaux, } \\
\text { sable, matière organique, sels solubles) liés } \\
\text { aux caractéristiques géologiques ou à la } \\
\text { contamination du site de prélèvement } \\
\text { Caractéristiques physiques de l'argile } \\
\text { inadaptées à la production de filtre du fait } \\
\text { d'une mauvaise plasticité ou consistance par } \\
\text { exemple. }\end{array}$ \\
\hline $\begin{array}{l}\text { Evaluation de la qualité par des } \\
\text { tests organoleptiques }\end{array}$ & $\begin{array}{l}\text { - Utilisation d'argile contenant des germes } \\
\text { et/ou impuretés chimiques pour la } \\
\text { production des filtres car la qualité et la } \\
\text { pureté de l'argile ne sont pas } \\
\text { rigoureusement évaluées par des tests de } \\
\text { Laboratoire }\end{array}$ \\
\hline $\begin{array}{l}\text { Traitement de la matière } \\
\text { première (exposition-séchage) }\end{array}$ & 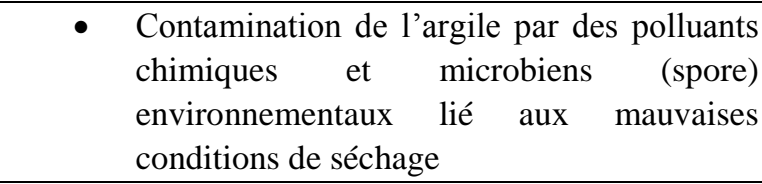 \\
\hline $\begin{array}{l}\text { Mélange des matières } \\
\text { premières : traitement } \\
\text { mécanique }\end{array}$ & $\begin{array}{l}\text { - Défaillance des filtres produits (débit de } \\
\text { filtration inadapté) par utilisation d'un } \\
\text { mauvais ratio de mélange argile/sciure de } \\
\text { bois liés à une insuffisance de savoir-faire } \\
\text { en poterie (main-d'œuvre artisanale) }\end{array}$ \\
\hline $\begin{array}{l}\text { Traitement à la chaleur : cuisson } \\
\text { des pots }\end{array}$ & $\begin{array}{l}\text { - Mauvaise cuisson des pots en céramique } \\
\text { (altération/survie des spores microbiens) due } \\
\text { à une température de cuisson non réglée } \\
\text { (cuisson dans un four en terre au feu de } \\
\text { bois) }\end{array}$ \\
\hline
\end{tabular}


Tableau 3 : Principaux dangers identifiés et mesures de prévention proposées.

\begin{tabular}{ll}
\hline DANGERS IDENTIFIES & MESURES PREVENTIVES \\
\hline $\begin{array}{l}\text { Choix de l'argile } \\
\text { - Présence de germes pathogènes (bactéries, } \\
\text { virus, champignons) }\end{array}$ & - $\begin{array}{l}\text { Réaliser un diagnostic } \\
\text { environnemental préalable au choix } \\
\text { du site de prélèvement }\end{array}$ \\
- Présence d'impuretés chimiques (métaux, \\
sable, matière organique, sels solubles) \\
Caractéristiques physiques de l'argile \\
inadaptées à la production de filtre
\end{tabular}

Traitement de la matière première (expositionséchage)

- Contamination de l'argile par des polluants chimiques et microbiens (spore) environnementaux lié aux mauvaises conditions de séchage

- Maintenir la zone de séchage propre et à l'abri de l'humidité et vérifier que les produits sont effectivement secs.

\section{Mélange des matières premières : traitement mécanique}

- Défaillance des filtres produits (débit de filtration inadapté)
- Renforcer les compétences du personnel en savoir-faire en poterie

- Déterminer et respecter un ratio de mélange argile/sciure de bois adéquat

- $\quad$ Faire recourt à un four à température réglable ou automatisé ;

- Surveiller la température et la durée de cuisson

Tableau 4 : Points critiques de contrôle de la chaîne de production du FILTRAO ${ }^{\circledR}$.

\begin{tabular}{|c|c|c|c|c|c|}
\hline $\begin{array}{l}\text { QUESTIONS } \\
\text { ETAPES }\end{array}$ & $\mathbf{Q}_{1}$ & $\mathbf{Q}_{2}$ & $\mathbf{Q}_{3}$ & $\mathbf{Q}_{4}$ & CCP? \\
\hline Choix de l'argile & OUI & $\mathrm{NON}$ & OUI & OUI & NON \\
\hline $\begin{array}{l}\text { Evaluation de la qualité de } \\
\text { l'argile }\end{array}$ & OUI & OUI & NA & NA & CCP1 \\
\hline $\begin{array}{l}\text { Traitement de la matière } \\
\text { première } \\
\text { séchage) }\end{array}$ & OUI & NON & OUI & OUI & NON \\
\hline $\begin{array}{ll}\begin{array}{l}\text { Mélange des matières } \\
\text { premières : }\end{array} & \text { traitement } \\
\text { mécanique } & \end{array}$ & OUI & OUI & NA & NA & $\mathrm{CCP} 2$ \\
\hline Traitement à la chaleur & OUI & OUI & NA & NA & CCP3 \\
\hline
\end{tabular}




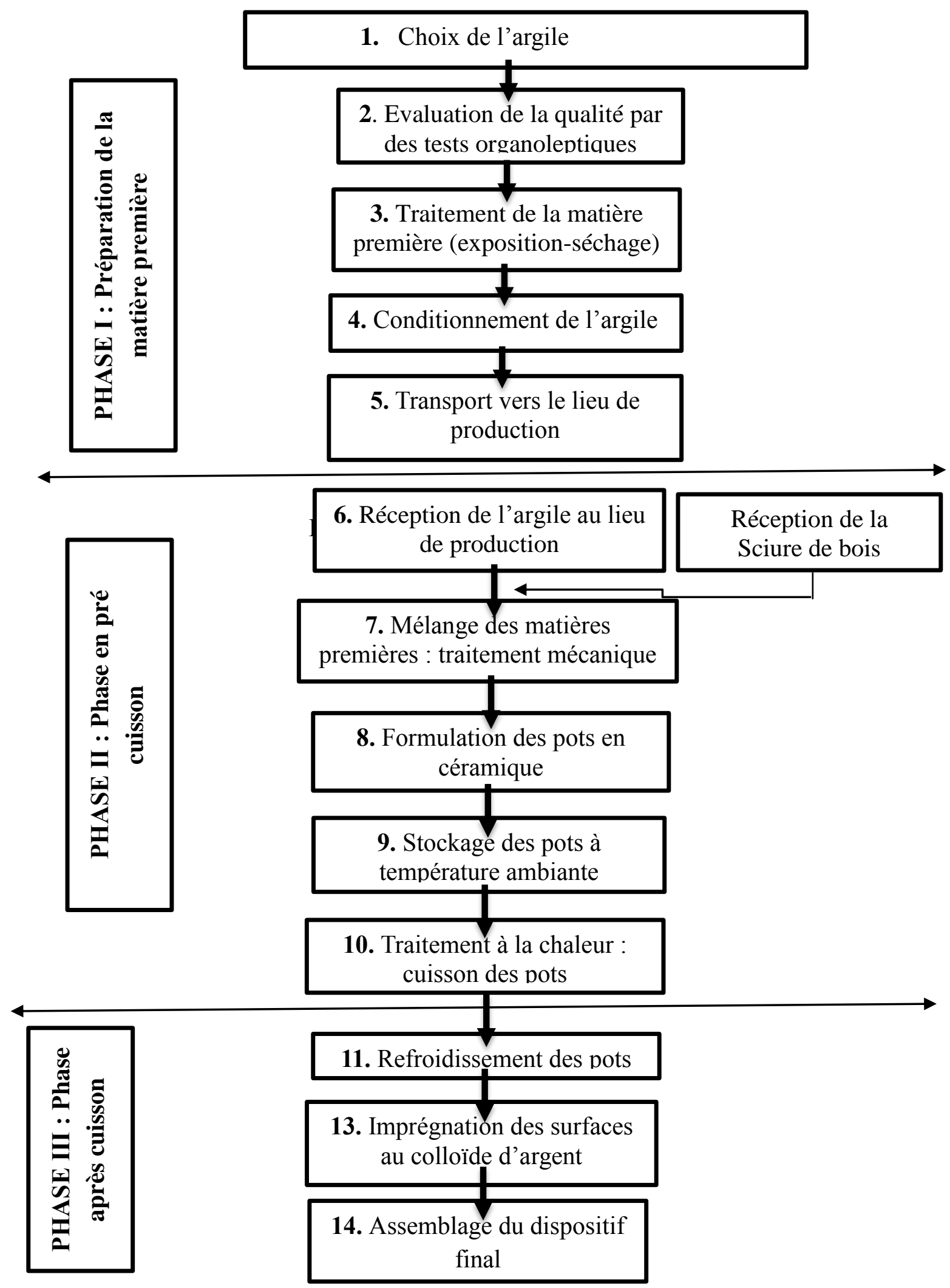

Figure 3 : Diagramme de fabrication du FILTRAO $^{\circledR}$. 


\section{DISCUSSION}

Le traitement de l'eau au point d'utilisation, peut constituer pour les pays en développement, une option prometteuse pour assurer l'accès à l'eau potable aux populations qui en sont dépourvues, mais aussi accélérer les gains sanitaires liés aux sources améliorées d'approvisionnement en eau. La garantie de l'efficacité et de l'innocuité des dispositifs utilisés à cet effet est un préalable à l'atteinte de tels objectifs. Ces derniers doivent par conséquent se conformer à des standards rigoureux de production et d'assurance qualité.

$\mathrm{Au}$ cours de cette étude, les principes de l'approche HACCP ont été appliqués au management de la sécurité sanitaire des filtres en céramique FILTRAOß. Les résultats obtenus révèlent que la production de ces filtres se fait en 14 étapes pouvant être subdivisées en 3 phases notamment la phase de préparation de la matière première, la phase en pré cuisson et la phase après cuisson. Les principaux points critiques de contrôle ont été identifiés aux étapes d'évaluation de la qualité de l'argile, de traitement mécanique des matières premières et de cuisson des pots en céramiques. En effet, le prélèvement de l'argile se faisait de manière aléatoire au gré des prospections et l'évaluation de sa qualité se résumait à des tests organoleptiques portant sur l'aspect général et le goût. Or l'argile prélevée dans l'environnement peut contenir divers polluants chimiques (métaux) et microbiologiques susceptibles d'être relargués par le filtre au cours de son utilisation (Van et al., 2007; Archer et al., 2011). De même, Vinka et collaborateur (2008) ont montré à l'issue d'une étude portant évaluation de filtres en céramiques produits à base de différentes terres argileuses que les caractéristiques de l'argile influencent le débit de filtration mais aussi les performances épuratoires des filtres. Ainsi donc, la qualité de l'argile, principale matière première entrant dans la composition du FILTRAO ${ }^{\circledR}$ devrait faire objet d'une évaluation rigoureuse pour optimiser ses performances et garantir sa sécurité.
Le traitement mécanique des matières premières est la seconde étape critique identifiée dans la chaîne de production du FILTRAO®. Cette étape regroupe un ensemble d'opérations dont le mélange des matières premières que sont l'argile, la sciure de bois (matière organique combustible) et l'eau. Le ratio de mélange argile/sciure de bois n'était pas correctement déterminé et variait d'un filtre à un autre. Ce ratio est cependant déterminant pour l'obtention de filtres résistants à la chaleur lors de la cuisson au four (Elodie, 2014). En outre, l'aspect poreux du filtre lui est conféré par l'élimination de la matière organique pendant la cuisson. Dans ces conditions, la proportion de sciure de bois dans le mélange influence les caractéristiques des pores partant le débit de filtration et la capacité épuratoire du filtre (Elodie, 2014 ; Soppe et al., 2015).

La cuisson des filtres FILTRAO® est la dernière étape critique mise en évidence lors de cette étude. Elle était réalisée dans un four artisanal utilisant comme combustible le bois. La température de cuisson était difficilement réglable d'où le risque d'une mauvaise cuisson des pots céramique et d'une survie des pathogènes éventuels (spores microbiens).

Ainsi, les paramètres critiques identifiés au cours de ce travail font bien partie de ceux décrits dans la littérature comme influençant la qualité et la sécurité des filtres en céramiques (The Ceramics Manufacturing Working Group, 2010 ; Soppe et al., 2015). Ces résultats démontrent donc que la démarche HACCP peut être utilisée avec succès pour la maitrise des risques sanitaires liés à la production des filtres en céramique FILTRAO ${ }^{\circledR}$ et cela bien que l'ensemble des étapes de la démarche HACCP n'ont pu être mis en œuvre et que tous les éléments de la chaîne de production n'ont pu être pris en compte dans l'analyse des dangers, faute d'une équipe HACCP expérimentée et disponible au sein de l'unité de production. Ces résultats préliminaires révèlent néanmoins que la production du FILTRAO ${ }^{\circledR}$ se fait selon un procédé artisanal susceptible de compromettre la sécurité et 
l'innocuité du produit fini. L'amélioration du procédé par une optimisation des étapes critiques mais aussi l'adoption effective d'une démarche d'assurance qualité telle l'approche HACCP applicable à l'ensemble de la chaîne de production sont un minimum de pré-requis pour garantir la fiabilité de ces dispositifs.

\section{Conclusion}

Cette étude s'était proposée d'identifier, les paramètres critiques dans la production des filtres en céramique FILTRAO®. Il en ressort que ces filtres sont fabriqués selon un procédé artisanal au cours duquel trois paramètres majeurs semblent influencer la qualité du produit fini à savoir, la qualité de l'argile, le ratio de mélange argile/sciure de bois et la température de cuisson. L'amélioration de ce procédé par l'adoption et l'application effective d'une démarche qualité s'avère nécessaire. $\mathrm{La}$ création d'une équipe HACCP et surtout l'implémentation de l'ensemble des étapes de la démarche HACCP pourraient contribuer à l'optimisation du procédé de fabrication et à l'atteinte d'une assurance qualité pour le FILTRAO®.

\section{CONFLITS D'INTERETS}

Les auteurs déclarent qu'il n'existe aucun conflit d'intérêts en ce qui concerne cet article.

\section{CONTRIBUTIONS DES AUTEURS}

LKK a conçu le protocole d'étude. BMCO, MBG et JKS ont collecté et analysé les données. MBG, JKS et JSC ont contribué à la rédaction de l'article.

\section{REMERCIEMENTS}

Les auteurs adressent leurs remerciements sincères aux membres de l'Association N Christ, à la direction de l'INHP et au personnel du Laboratoire d'Hygiène de l'INHP.

\section{REFERENCES}

Annette P-U, Jamie B, Thomas C, John MCJ, Oliver C, Valerie C, Sophie B, Alan DD, Jennifer De F, Lorna F, Matthew CF,
Bruce G, Paul RH, Richard BJ, Colin M, Daniel M, Kate M, Maria N, Meredith S, Jennyfer W, Sandy C. 2014. Burden of disease from inadequate water, sanitation and hygiene in low-and middle-income settings: a retrospective analysis of data from 145 countries. Tropical Medicine \& International Health, 19(8): 894-905. DOI: $10.1111 /$ tmi.12329.

Aminata K, Boubacar S, Almamy K, Jacques S, Paul W, Brigitte IK, Lambert ZPN, Dayéri D. 2018. Impact of chlorination on the microbiological quality of drinking water in school area: case study of Central North Region in Burkina Faso. Int. J. Biol. Chem. Sci., 12(3): 1225-1233.

DOI: https://dx.doi.org/10.4314/ijbcs.v12i3.12

Amroun HKF. 2017. La contribution à l'installation de la démarche HACCP dans une chaine de fabrication d'une boisson à base de concentré d'orange TAIBA FOOD COMPANY. Master en Génie des Procédés, Université M'hamed Bougara Boumerdes Algérie, Boumerdes, p.100.

Archer AR, Elmore AC, Bell E, Rozycki C. 2011. Field investigation of arsenic in ceramic pot filter-treated drinking water. Wat. Sci. Tech., 63(10): 2193-2198. DOI: $10.2166 /$ wst.2011.406

Babafemi AA, Yinusa DL. 2015. Formulation of Ceramic Water Filter Composition for the Treatment of Heavy Metals and Correction of Physiochemical Parameters in Household Water. Art and Design Review, 3: 94-100. DOI: http://dx.doi.org/10.4236/adr.2015.34013 Bain R, Cronk R, Wright J, Yang H, Slaymaker T, Bartram J. 2014. Fecal Contamination of Drinking-Water in Low- and Middle-Income Countries: A Systematic Review and Meta-Analysis. PLoS Med, 11(5) : 23p. DOI : 10.1371/journal.pmed.1001644 
Boudjelti ML. 2017. Contribution à la mise en place de la démarche HACCP dans la chaine de production des plats cuisinés chauds au niveau du Catering AIR ALGERIE. Master en Genie des Procédés, Université M'hamed Bougara Boumerdes Algérie, Boumerdes, p. 138.

Codex Alimentarius Commission 2003. Code d'usages international recommandé Principes généraux d'hygiène alimentaire - CAC/RCP 1-1969, Rév. 4 (2003).

Dewettinck T, Van Houtte E, Geenens D, Van Hege K, Verstraete W. 2001. HACCP (hazard analysis and critical control points) to guarantee safe water reuse and drinking water production-a case study. Wat. Sci. Tech., 43(12): 31-38. DOI : 10.2166/wst.2001.0708

Elodie BB. 2014. Traitement de l'eau à domicile : Conception et évaluation de l'efficacité des filtres en céramique. Mémoire Master Ingénierie de l'Eau et de l'Environnement, Institut International d'Ingénierie de l'Eau et de l'Environnement Burkina Faso, Ouagadougou, p. 64.

Erhuanga E, Kashim IB, Akinbogun TL. 2014. Development of Ceramic Filters for Household Water Treatment in Nigeria. Art and Design Review, 2(1): 610.

DOI:

http://dx.doi.org/10.4236/adr.2014.21002

Espérance OH, Micheline ADT, Nelly CK, Expédit WV, Guy AM, Euloge A. 2016. Pollution des eaux à usages domestiques dans les milieux urbains défavorisés des pays en développement : Synthèse bibliographique. Int. J. Biol. Chem. Sci., 10(5): 2392-2412. DOI: http://dx.doi.org/10.4314/ijbcs.v10i5.35

Hunter, PR. 2009. Household water treatment in developing countries: comparing different intervention types using metaregression, Environ. Sci. Technol., 43(23): $\quad$ 8991-8997. DOI: 10.1021/es9028217.
Jagals C, Jagals P. 2004. Application of HACCP principles as a management tool for monitoring and controlling microbiological hazards in water treatment facilities, Water Science and Technology, 50(1): 69-76. $\quad$ DOI: 10.2166/wst.2004.0022

Danièle SL, Robert Q, Eric DM. 2006. Household water treatment and safe storage options in developing countries: a review of current implementation practices. Wilson Quarterly, Woodrow Wilson International Center for Scholars Environmental Change and Security Program, Washington, p.143.

Mark DS, Christine ES, Lisa MC, Joseph MB, Mark AE. 2008. Point of Use Household Drinking Water Filtration: A Practical, Effective Solution for Providing Sustained Access to Safe Drinking Water in the Developing World. Environmental Science and Technology, 42(12): 42614267. DOI http://dx.doi.org/10.1021/es702746n

Moubokounou GL. 2010. Techniques de Traitement de l'Eau à Domicile : Evaluation de l'efficacité des Filtres En céramique. Mémoire Master Ingénierie de L'eau et de L'environnement, Institut International d'Ingénierie de l'Eau et de l'Environnement Burkina Faso, Ouagadougou, p. 67.

Murphy HM, McBean EA, Khosrow F. 2010. A critical evaluation of two point-of-use water treatment technologies: can they provide water that meets $\mathrm{WHO}$ drinking water guidelines. Journal of Water and Health, 8(4): 20p. DOI: 10.2166/wh.2010.156.

Njoyim EBT, Nchofua FB, Mofor NA, Djoko YT. 2016. Contrôle de la qualité des eaux domestiques dans le village Babessi au Nord-Ouest Cameroun. Int. J. Biol. Chem. Sci., 10(3) : 1382-1402. DOI : http://dx.doi.org/10.4314/ijbcs.v10i3.37 
OMS. 2011. Stratégies pour la gestion sans risque de l'eau de boisson destinée à la consommation humaine. SoixanteQuatrième Assemblée Mondiale de la Santé, Genève, Point 13.15, document A64/24, p.7.

Robert N, James O-O. 2015. Evaluating coagulant activity of locally available Syzygium cumini, Artocarpus heterophyllus and Moringa oleifera for treatment of community drinking water, Uganda. Int. J. Biol. Chem. Sci., 9(6): 2535-2554.

DOI: http://dx.doi.org/10.4314/ijbcs.v9i6.3.

Soppe AIA, Heijman SGJ, Gensburger I, Shantz A, Van Halem D, Kroesbergen J, Wubbels GH, Smeets PWMH. 2015. Critical parameters in the production of ceramic pot filters for household water treatment in developing countries. Journal of Water and Health, 13(2): 587599 DOI: 10.2166/wh.2014.090.

The Ceramics Manufacturing Working Group. 2010. Best Practice Recommendations for Local Manufacturing of Ceramic
Filters for Water Treatment, Ed. 1. Seattle, WA, USA.

Van Halem D, Heijman SGJ, Soppe AIA, Van Dijk JC, Amy GL. 2007. Ceramic silverimpregnated pot filters for household drinking water treatment in developing countries: material characterization and performance study. Water Science and Technology: Water supply, 7(5-6): 9-17. DOI: $10.2166 /$ ws.2007.142

Vinka A, Oyanedel-Craver, James AS. 2008. Sustainable Colloidal-SilverImpregnated Ceramic Filter for Point-ofUse Water Treatment. Environmental Science and Technology, 42(3): 927-933. DOI: $10.1021 /$ es071268u. 